
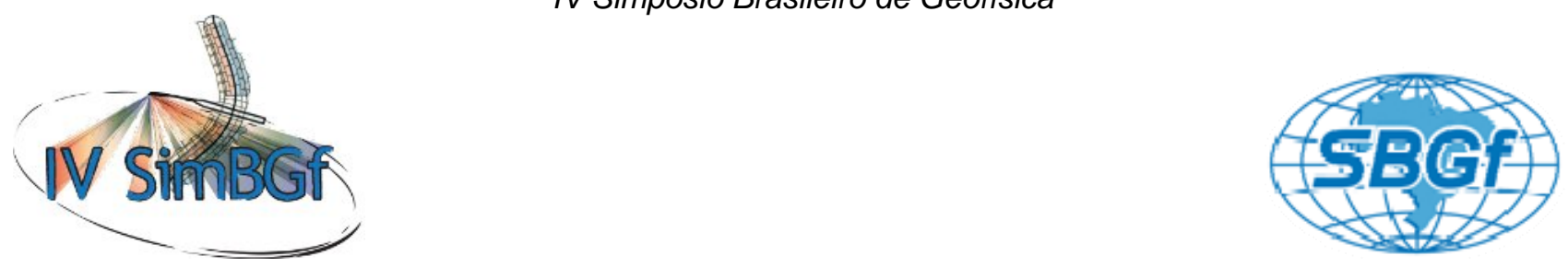

\title{
Identificação de Depósitos de Fosfatos na região de Catalão, Goiás, por meio de Dados Termais do Sensor ASTER
}

Renata de Araújo Teixeira, Gustavo Macedo de Mello Baptista, Paulo Roberto Meneses, Instituto de Geociências Universidade de Brasília

Copyright 2008, SBGf - Sociedade Brasileira de Geofísica

Este texto foi preparado para a apresentação no IV Simpósio Brasileiro de Geofísica, Belém, 14 a 17 de novembro de 2010. Seu conteúdo foi revisado pelo Comitê Técnico do IV SimBGf, mas não necessariamente representa a opinião da SBGf ou de seus associados. É proibida a reprodução total ou parcial deste material para propósitos comerciais sem prévia autorização da SBG.

\section{Resumo}

O presente estudo foi realizado com intuito de testar a eficiência das imagens termais do sensor ASTER para prospecção de depósitos de fosfato. A região de estudo faz parte de um grande complexo carbonatítico alcalino, cuja rocha geradora de fosfato é chamada de foscorito e o mineral característico dessas rochas é essencialmente a apatita, que ocorre associada aos minerais de magnetita e a flogopita, principalmente. Com base nessas informações foi aplicada a técnica de classificação espectral Spectral Feature Fitting - SFF, utilizando-se como endmember o espectro de emissividade 501 de apatita da Biblioteca Espectral da Arizona State University que recobre a faixa do espectro termal de 5 a $45 \mu \mathrm{m}$. Por meio dessa classificação foi possível determinar a feição espectral da apatita na área de estudo, ressaltando na imagem as áreas propicias a prospecção de fosfato.

\section{Introdução}

A utilização de dados de sensoriamento remoto em conjunto com técnicas de processamento digital sempre teve importância nos estudos geológicos, principalmente na tentativa de identificação de feições espectrais de minerais, devido à minimização de custos e de tempo em campanhas de pesquisa e exploração.

Porém, o enfoque quase sempre estava relacionado aos sistemas que imageam o espectro óptico refletido ou nos sistemas que se dedicam à obtenção de informações nos espectros das microondas.

O presente estudo buscou uma compreensão em outra região do espectro eletromagnético, a faixa emitida do infravermelho termal. Para tal, foi utilizada uma cena do sensor ASTER (acrônimo de Advanced Spacerborne Thermal Emission Reflection Radiometer), que se encontra como carga útil do satélite Terra (EOS-AM1). O ASTER possui 3 subsistemas (VNIR - Visible Near Infrared; SWIR - Short Wave Infrared; e TIR - Thermal Infrared), sendo o TIR utilizado neste estudo.

O subsistema TIR opera em 5 bandas na janela atmosférica de 8 a $12 \mu \mathrm{m}$, na região do infravermelho emitido ou termal, com apenas um único telescópio de posição fixa com visada nadiral, com uma resolução espacial de $90 \mathrm{~m}$ e radiométrica de 12 bits (Jensen, 2009), conforme detalha a tabela 1.
Tabela 1. Características do subsistema TIR do ASTER.

\begin{tabular}{cccc}
\hline Banda & $\begin{array}{c}\text { Comprimento } \\
\text { de onda }(\boldsymbol{\mu m})\end{array}$ & $\begin{array}{c}\text { Resolução } \\
\text { Espacial }\end{array}$ & $\begin{array}{c}\text { Resolução } \\
\text { Radiométrica }\end{array}$ \\
\hline 10 & $8,125-8,475$ & & \\
11 & $8,475-8,825$ & & 12 bits \\
12 & $8,925-9,275$ & $90 m$ & \\
13 & $10,25-10,95$ & & \\
14 & $10,95-11,65$ & & \\
\hline
\end{tabular}

O objetivo deste estudo foi a identificação dos foscoritos e de rochas fosfatadas, por meio das imagens termais do ASTER, localizadas na região de Catalão, ao sul do estado de Góias (Fig. 1).

O complexo ultramáfico-carbonatítico-alcalino de Catalão I é um dos vários complexos alcalinos mesozóicos, que se encontra nas bordas da Bacia do Paraná, limitado por falhas profundas na direção NW (REQUEIJO e MONTAVANI, 2008).

Esse complexo ocupa uma área de cerca de 26 milhões $\mathrm{m}^{2}$ e possui uma estrutura na forma de um platô subcircular, com eixos NW (6km) e NE (5km), sustentado por rochas quartzíticas.

O complexo é composto por carbonatitos afetados por metassomatismo com geração de foscoritos e glimeritos, nos quais as intrusões produziram auréolas de fenetização resultantes da reação entre as soluções ígneas, rica em álcalis e alumina, e os xistos aluminosos do Grupo Araxá, o que resultou em feldspatização, nefelinização e aereginização, brechação e pegmatização (CORDEIRO, 2009).

Tal complexo hospeda uma diversidade de rochas e mineralizações, dentre elas, os foscoritos, que são rochas ígneas intrusivas raras, cuja mineralogia é composta principalmente por apatita, magnetita, diopsidio forsterita ou flogopita (WALL e ZAITSEV, 2004).

Os foscoritos que fazem parte desse complexo são encontrados principalmente na forma de um material terroso saprolitizado, derivado do processo de intemperismo. Essas rochas são o foco deste trabalho, já que as mesmas comportam depósitos de fosfatos, industrializados pela empresa de mineração Fosfértil. 


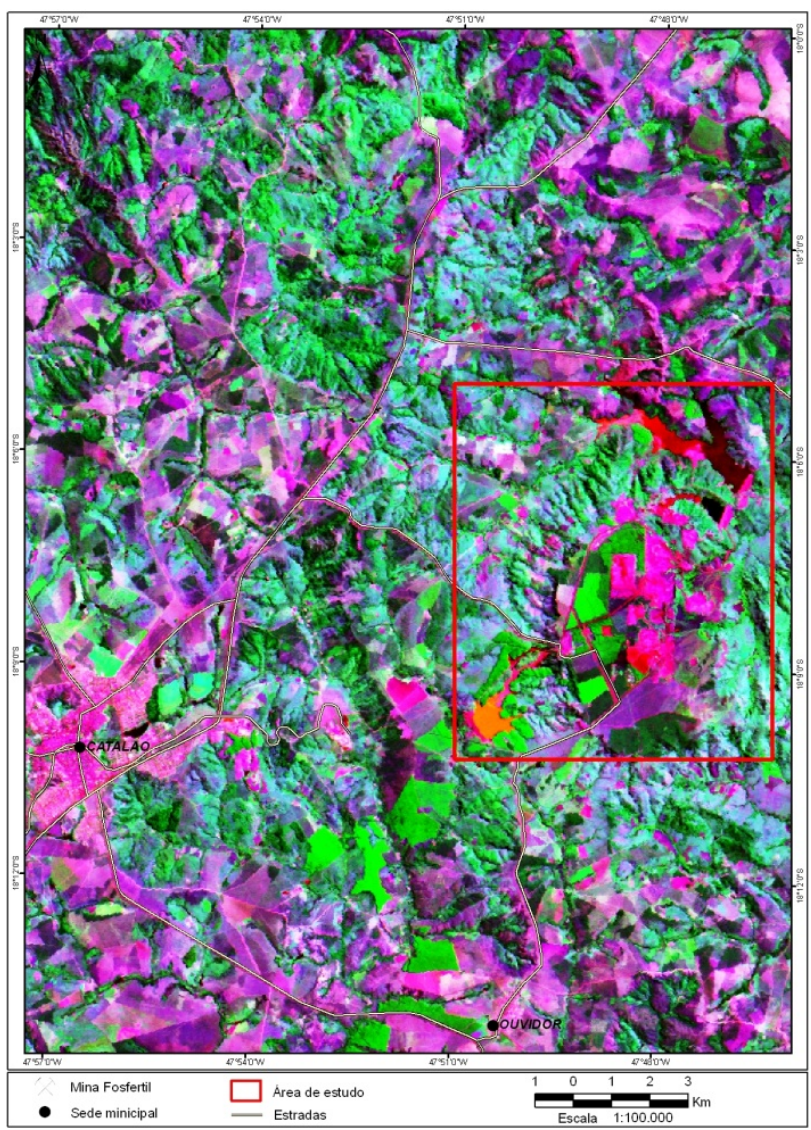

Figura.1. Localização da área de estudo na imagem Landsat TM.

\section{Material e Métodos}

Para a realização deste estudo utilizou-se o AST-05 produto sob demanda de emissividade de superfície, estimada pelo método de emissividade normalizada e é iterativamente compensado pela luz solar refletida. O espectro de emissividade é normalizado por meio da emissividade média de cada pixel. A diferença mínima e máxima (MMD) do espectro normalizado é calculada e as estimativas da emissividade mínima são obtidas por meio de análise de regressão. Essas estimativas são usadas para reescalonar a emissividade normalizada e para compensar a reflexão da luz do céu como forma de aperfeiçoamento de emissividade. Além disso, esse produto é disponibilizado com correção para os efeitos atmosféricos de absorção e espalhamento.

Para o processamento da imagem termal utilizou-se o software ENVI $4.7 \AA$, dividindo-o em duas etapas: préprocessamento e processamento.

O pré-processamento envolveu técnicas básicas de junção de bandas em um único arquivo e georreferenciamento da imagem AST-05 e conversão dos dados brutos para emissividade por meio do fator 0,001 . Além disso, foi necessária a construção da biblioteca espectral de emissividade da apatita, a partir dos espectros da Biblioteca da Arizona State University, que foram convertidos de número de onda para comprimento de onda, expressos em micrômetros, e depois reamostrados para as funções dos detectores do ASTER.

O processamento baseou-se na aplicação da técnica de classificação espectral Spectral Feature Fitting - SFF, na qual foram utilizados os espectros reamostrados. Essa técnica determina um ajuste de mínimos quadrados das feições espectrais do pixel em relação ao espectro de referência, ou da biblioteca espectral, chamado de endmember. Como resultado, duas imagens são obtidas, a scale que mostra o ajuste entre as feições do pixel e do endmember, e a RMS é representa o erro médio quadrático do ajuste. Ao se dividir a imagem scale pela RMS pode-se obter um terceiro produto, a fit image, que pode ser entendida como a probabilidade de o pixel apresentar o endmember.

Nos resultados do classificador SFF foram aplicados filtros de passa baixa com operador $5 \times 5$, atribuída uma tabela de cores (rainbow) e foram ajustados os histogramas visando realçar as áreas tanto na imagem scale, como de ruído, na RMS.

Após o processo de otimização da identificação das áreas, realizou-se uma fusão do tipo RGB-IHS entre o resultado da imagem scale e a banda 3 ASTER $(0,76$ $0,86 \mu \mathrm{m}$ ) com $15 \mathrm{~m}$ de resolução espacial.

Visando verificar a eficiência do processamento dos dados termais, foi realizada, na semana de 4 a 10 de julho de 2010, uma visita de campo a mina a céu aberto de Catalão.

\section{Resultados e Discussão}

O processamento das imagens do sensor - TIR (ASTER) permitiu à identificação dos locais mais propícios a ocorrência dos depósitos de fosfatos, os quais foram confirmados na visita de campo. O fato de o depósito encontrar-se em mina a céu aberto facilitou a identificação do endmember de apatita da biblioteca espectral do ASU.

A biblioteca espectral da Universidade do Arizona (ASU) destaca, para apatita, feições espectrais com picos centrados a 9,1 $\mu \mathrm{m}$, conforme a figura 2 .

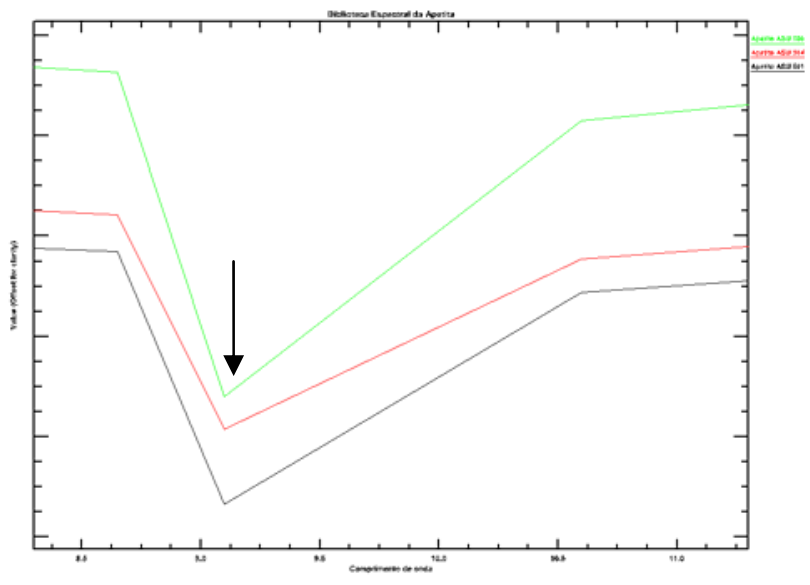

Figura 2. Biblioteca espectral da Apatita.

Como resultado da aplicação da metodologia descrita, obteve-se uma imagem scale da classificação pelo algoritmo SFF, que corresponde aos pixels que 
apresentam maior intensidade de feição espectral associada ao mineral apatita (fig.3).

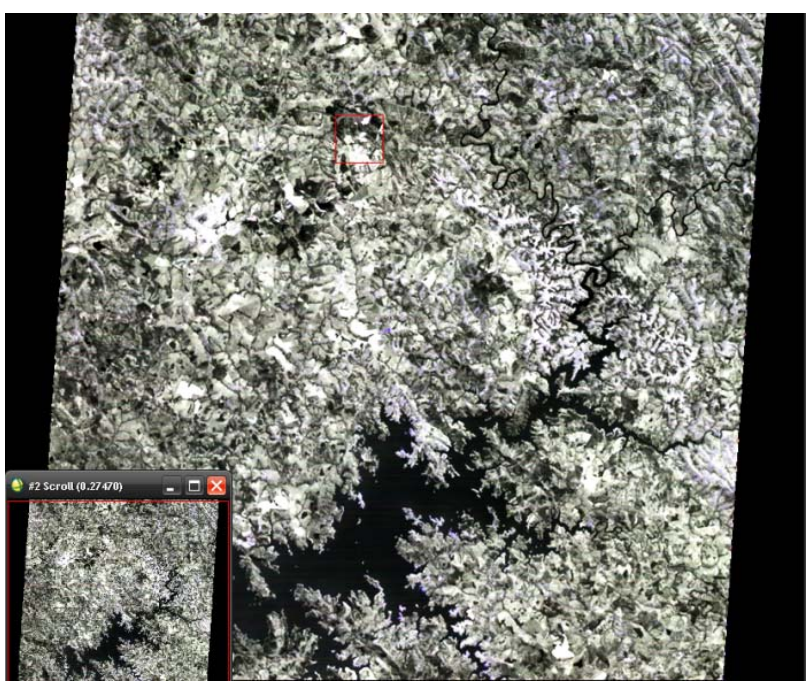

Figura 3. Imagem scale obtida para área de estudo.

Ao otimizar o resultado da imagem SFF, por meio de filtragem e de expansão histogrâmica, a classificação ressaltou a resposta espectral da apatita na imagem, na qual, na escala de cores, os valores mais elevados que apresentam maior intensidade da feição espectral são respresentados como os mais vermelhos, e portanto, correspondem as regiões mais propicias a ocorrência dos depósitos de fosfatos (fig.4).

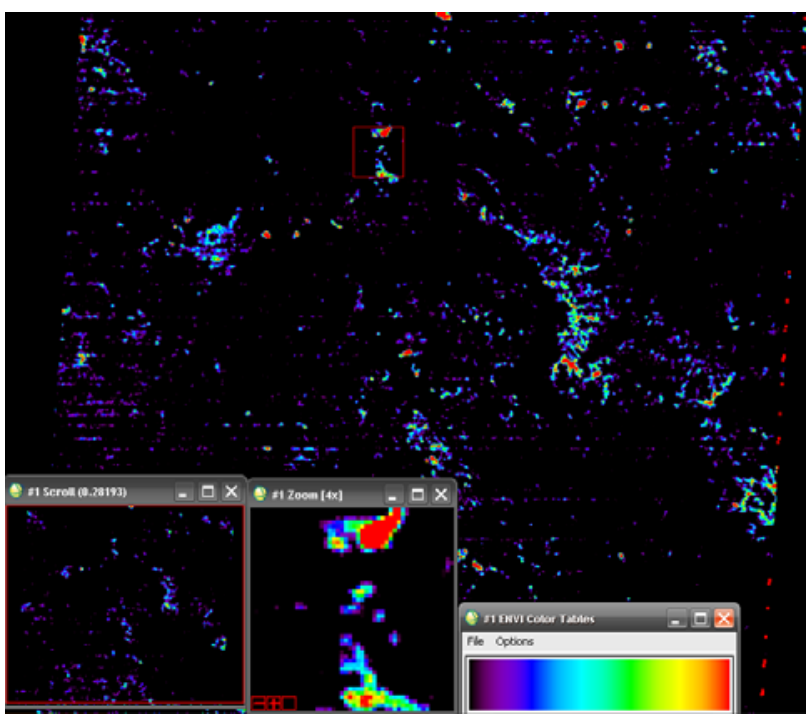

Figura 4. Imagem scale destacando as áreas com maiores valores relativos ao endmember de apatita em vermelho.

A classificação espectral apresentou bons resultados para a região do complexo carbonatítico de Catalão.

Para ressaltar isso, foi efetuada, ainda, uma fusão entre o resultado SFF com a imagem $3 \mathrm{~N}$ do sensor ASTER, que apresenta $15 \mathrm{~m}$ de resolução espacial, e representa a região do vermelho. O que resultou numa imagem de melhor resolução espacial com as áreas identificadas no SFF como mais propicias para a ocorrência de fosfatos destacadas na cena (fig.5).

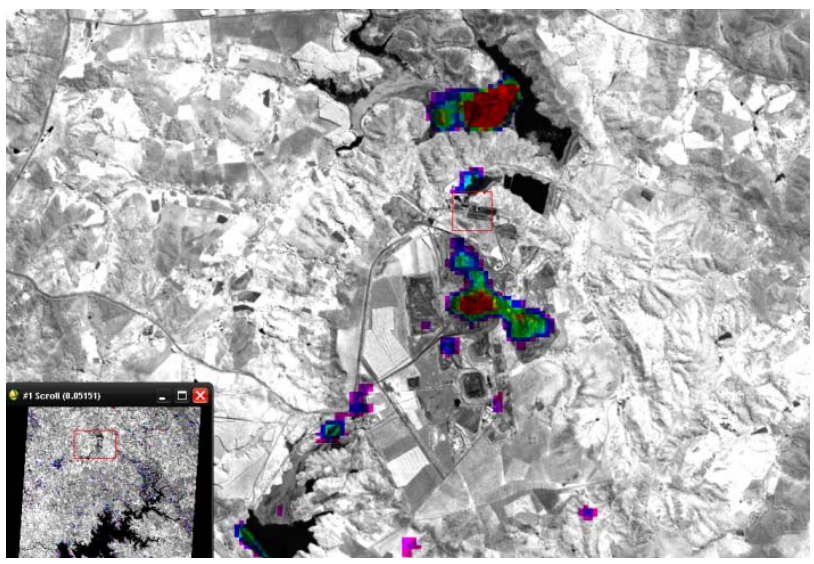

Figura 5. Fusão HSV destacando as áreas com maiores feições espectrais de apatita coincidentes com a mina a céu aberto.

Visando comprovar a eficácia da utilização de dados termais para estudos prospectivos de depósitos fosfáticos por meio da classificação SFF, buscou-se os espectros das áreas como os valores mais elevados e o que podese notar foi a mesmo formato de espectro obtido pela biblioteca espectral da ASU reamostrados para os dados ASTER (fig.6).

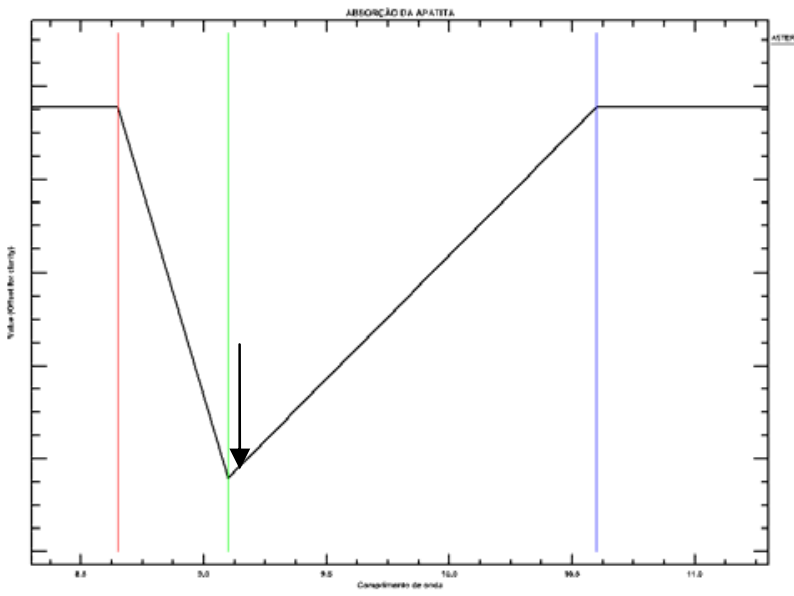

Figura 6. Feição espectral obtido na imagem scale Absorção da Apatita SFF (imagem ASTER)

\section{Discussão e Conclusões}

Os produtos ASTER sob demanda na porção do termal tem sido explorados com eficiência para feições espectrais minerais (HOOK et al., 2005).

A feição espectral de apatita, centrada a $9 \mu \mathrm{m}$, vem sendo investigada nas porções emitidas do espectro eletromagnético (CHRISTENSEN et al., 2000; LANE et al., 2007). 
O presente estudo permitu concluir que a aplicação da classificação espectral utilizando imagens ASTER no subsistema TIR para estudos de depósitos fosfáticos apresentam resultados bastante eficazes e esperansosos, principalmente depois da avaria ocorrida com o sensor SWIR em abril de 2008, que o tornou inoperante.

A aplicabilidade da metodologia proposta pode servir como norteadora em estudos prospectivos e de mapeamento geológico da área de investigação, mas também pode minimizar custos nas fases iniciais.

Por fim, os resultados obtidos motivam 0 desenvolvimento de novas investigações como forma de consolidar os estudos mineralógicos em espectros termais.

\section{Agradecimentos}

A Universidade de Brasília e a empresa de mineração Fosfértil, indispensáveis para realização desse estudo.

\section{Referências}

Christensen, P.R., Bandfield, J.L., Hamilton, V.E., Howard, D.A., Lane, M.D., Piatek, J.L., Ruff, S.W., \& Stefanov, W.L., 2000. A Thermal Emission Spectral Library of Rock-Forming Minerals. Journal of Geophysical Research (Planets), 105, 9735.

Cordeiro P. F. O.; Brod J. A., 2009. Petrologia e Metalogenia do Depósito Primário de Nióbio do Complexo Carbonatítico-fosforítico de Catalão I (GO). Instituto de Geologia. Univerdidade de Brasília. p. 14-17.

ESRI. 2006. ArcGis - Versão 9.2.

Hook, S. J., J. E. Dmochowski, K. A. Howard, L. C. Rowan and K. E. Karlstrom and J. M. Stock, 2005. Mapping variations in weight percent silica measured from multispectral thermal infrared imagery - Examples from the Hiller Mountains, Nevada, USA and Tres Virgenes-La Reforma, Baja California Sur, Mexico. Remote Sensing of Environment, vol. 95, pp. 273-289.

Jensen, J. R. 2009. Sensoriamento Remoto do Ambiente. Uma pespectiva em recursos terrestres. University of South Carolina. Editora Parêntese. p. 232-234.

Lane, M. D., Dyar, M. D., Bishop, J. L. 2007. Spectra of Phosphate Minerals as Obtained by Visible-Near Infrared Reflectance, Thermal Infrared Emission, and Mössbauer Laboratory Analyses. Lunar and Planetary Science XXXVIII.

Requeijo, H. S \& Mantavani, M. S. M. 2008.Gravimetria do Complexo Alcalino de Catalão I (GO): Análise Preliminar. Instituto de Astronomia Geofísica e Geociências. Universidade de São Paulo. IV Simpósio de Vulcanismo e Ambientes Associados. Foz do Iguaçu, Paraná. p. 2.
RSI (Research System Inc.). 2007. ENVI: Environment for Visualizing Images. Versão 4.7.

Wall, F. \& Zaitsev, A. N. (eds) 2004. Phoscorites and Carbonatites from Mantle to Mine: the Key Example of the Kola Alkaline Province. Cambridge University Press. Geological Magazine; v.143, p.140-141. 